\title{
Research on Dispersion Compensation of 40 GB/s Optical Duo-Binary Coded Transmission System
}

\author{
Yue Zhou, Yufeng Shao*, Zhifeng Wang*, Changxiang Li, Junyi Zhou, Wenzhe Ma \\ Faculty of Engineering, Shanghai Polytechnic University, Shanghai, China \\ Email: "yfshao@sspu.edu.cn, "zfwang@sspu.edu.cn
}

Received 1 July 2016; accepted 18 August 2016; published 25 August 2016

\begin{abstract}
A $40 \mathrm{~Gb} / \mathrm{s}$ high speed optical transmission system is designed, and the different dispersion compensation schemes are adopted to realize the transmission of the optical duo-binary (ODB) signals over $120 \mathrm{~km}$ optical fiber. Optical duo-binary signals are generated by using a precoder, two lowpass filters (LPF) and one mach-zehnder modulator (MZM). Through combination of dispersion compensation fiber (DCF) and single mode fiber (SMF) in the transmission link, four different dispersion compensation schemes (pre-compensation, post-compensation and hybrid compensation (pre+ post) and hybrid compensation (post+ pre)) were compared. Analyzing $Q$ factor, BER, eye diagrams and receiver sensitivity in several dispersion compensation schemes, the simulation results are: the cut-off frequency of LPF affects the transmission performance, and the hybrid compensation (post+ pre) scheme is the optimal dispersion compensation method.
\end{abstract}

\section{Keywords}

Optical Communication, Duo-Binary, Dispersion Compensation, Q Factor, Eye Diagrams

\section{Introduction}

The two development directions of optical fiber transmission system are high speed and long span, and the attenuation and dispersion have effects on optical signals transmission [1]. Especially, the dispersion of SMF has reached $16 \mathrm{PS} / \mathrm{nm} \cdot \mathrm{km}$, the distance of $40 \mathrm{Gbit} / \mathrm{s}$ signal can only be transmitted to $4 \mathrm{~km}$ without using dispersion compensation techniques [2]. Nowadays, the optical dispersion compensation methods mainly included: dispersion compensation fiber (DCF), fiber Bragg grating (FBG), virtually imaged phased array and planar waveguide technology [3]. DCF technology has been widely used since it has large compensation bandwidth, technology maturity and stable performance [4]. At the same time, in order to increase the dispersion tolerance, extend the repeating distance and improve the spectrum efficiency, the optical duo-binary (ODB) coding has been used. ODB modulation format has some advantages like high dispersion tolerance, high spectrum efficiency and high anti-nonlinear ability in high-speed optical fiber communication system. The ODB signal has also better trans-

\footnotetext{
${ }^{*}$ Corresponding authors.
}

How to cite this paper: Zhou, Y., Shao, Y.F., Wang, Z.F., Li, C.X., Zhou, J.Y. and Ma, W.Z. (2016) Research on Dispersion Compensation of 40 GB/s Optical Duo-Binary Coded Transmission System. Optics and Photonics Journal, 6, 190-195. 
mission capability for its spatial response characteristic according to the Nyquist's second criterion [5] [6]. In this paper, optical duo-binary signals are generated using low pass filter (LPF-ODB) is proposed. In order to compensating dispersion, four kinds of dispersion compensation schemes are designed and compared.

\section{Principle of Dispersion Compensation}

The dispersion of SMF in the 1550nmwavelength is 17 - 20 ps with positive dispersion slope. In order to achieve high speed, large capacity and long distance communication, it is necessary to use DCF in the optical fiber [7], thus the total dispersion of the whole optical fiber line is approximately zero [8]. Compared to the SMF, DCF has larger attenuation. It is necessary to add the amplifier like EDFA to compensate in the system [9]. According to the flexible permutation and combination of DCF, SMF and EDFA, four kinds of compensation schemes are used to design simulation model. As shown in Figures 1(a)-(d) respectively, pre-compensation and post-compensation, hybrid compensation (pre+ post) and hybrid compensation (post ${ }^{+}$pre). The compensation method of simulation is symmetrical compensation, and the dispersion and dispersion slope of SMF can be compensated by DCF completely. The gain of EDFA after the SMF and DCF is $5 \mathrm{~dB}$ and $10 \mathrm{~dB}$ respectively, just might compensate to the attenuation of SMF and DCF. Parameters of the optical fiber are shown in Table $\mathbf{1}$.

\section{Results}

In this paper, Optisystem 13 is used to simulate a rate of $40 \mathrm{~GB} / \mathrm{s}$ dispersion compensation fiber optical transmission system. The system diagram is shown in Figure 2.

First of all, according to Figure 2, TX is consist of continuous wave (CW) laser, MZM, pseudo random sequence generator, non-return to zero(NRZ) pulse generator, binary pre coder and low-pass filter (LPF). The output wavelength of CW laser is $1550 \mathrm{~nm}$, the power is $3 \mathrm{dBm}$ and line width is $0.1 \mathrm{MHz}$. The extinction ratio

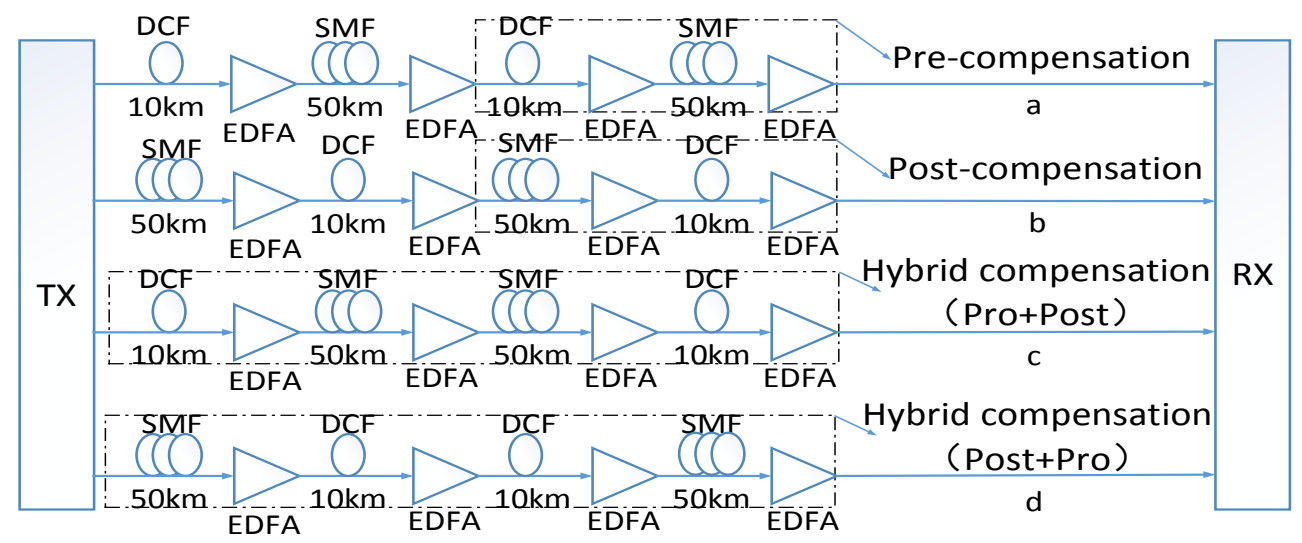

Figure 1. Dispersion compensation schemes (a) Pre-compensation (b) Post-compensation (c) Hybrid-compensation (Pre + Post) (d) Hybrid-compensation (Post+ Pre).

Table 1. Parameters of optical fiber link.

\begin{tabular}{ccc}
\hline Parameters & Single mode fiber & Dispersion compensating fiber \\
\hline Length $(\mathrm{km})$ & 50 & -80 \\
Dispersion $(\mathrm{ps} / \mathrm{nm} / \mathrm{km})$ & 16 & 30 \\
Dispersion slope $\left(\mu \mathrm{m}^{2}\right)$ & 80 & -20 \\
First order dispersion coefficient $\left(\mathrm{ps}^{2} / \mathrm{km}\right)$ & -20 & 0.5 \\
Attenuation $(\mathrm{dB} / \mathrm{km})$ & 0.2 & $2.6 \times 10^{-20}$ \\
Nonlinear refractive index $\left(\mathrm{m}^{2} / \mathrm{W}\right)$ & $2.6 \times 10^{-20}$ & -0.45 \\
Dispersion slope $\left(\mathrm{ps} /\left(\mathrm{nm}^{2} \mathrm{~km}\right)\right)$ & 0.075 & \\
\hline
\end{tabular}




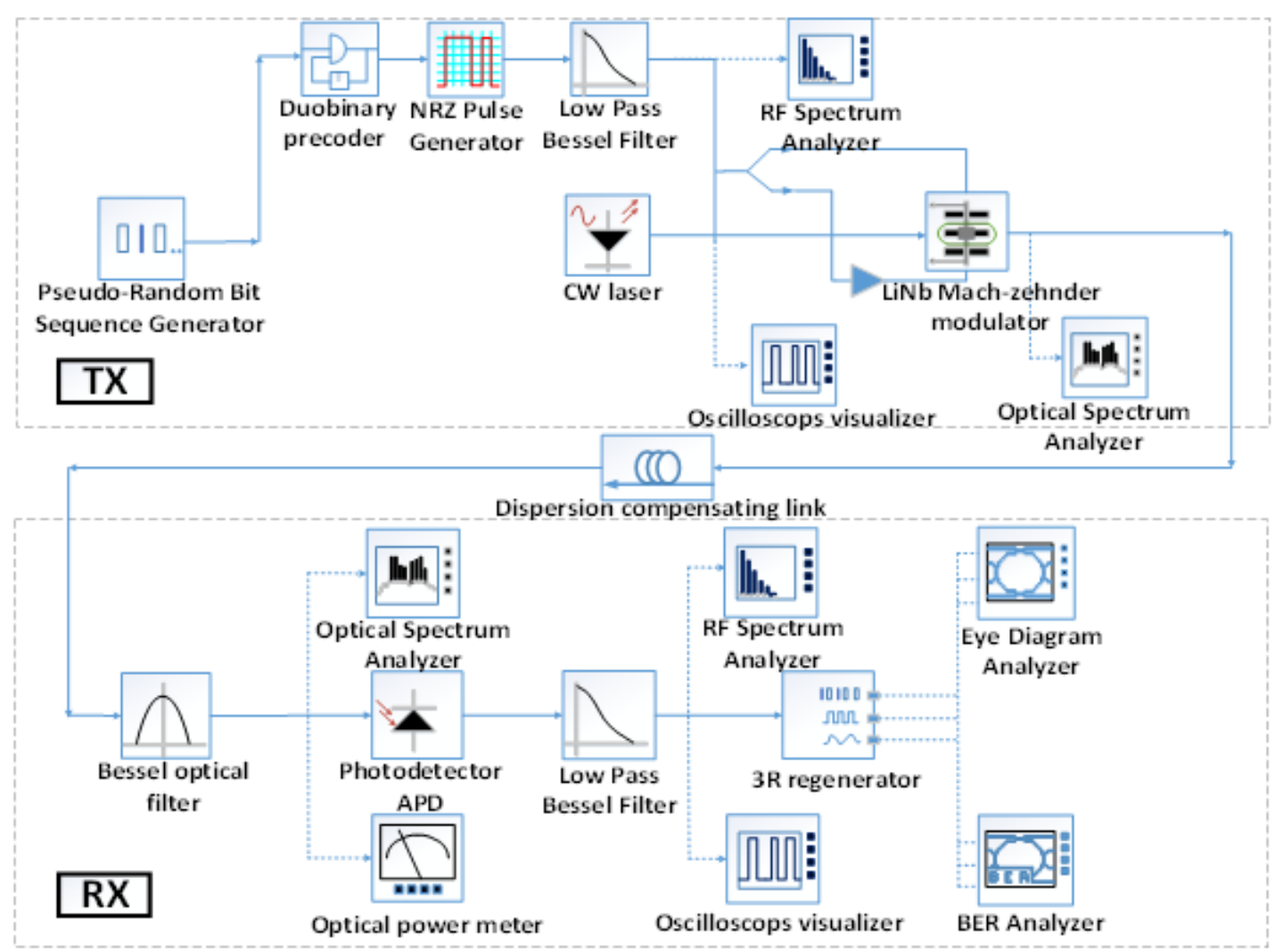

Figure 2. Transmission system diagram.

of MZM is $25 \mathrm{~dB}$, the insertion loss is $2 \mathrm{~dB}$ and the bias voltage is $4 \mathrm{~V}$; the cut-off frequency of low-pass filter is $15 \mathrm{GHz}$. Duo-binary modulation signals is externally modulated into MZM eventually. Under the push-pull mode, the electric signal will be transformed to optical duo-binary signal. In the optical fiber transmission, the length of SMF and DCF are $100 \mathrm{~km}$ and $20 \mathrm{~km}$ respectively according to the Formula (1). RX is mainly composed of low-pass Bessel optical filter, PIN photodiode, 3R regenerator and LPF, as shown in Figure 2. The bandwidth of low-pass Bessel optical filter is 1.75 bit rate, the response degree is $0.9 \mathrm{~A} / \mathrm{W}$ and the dark current is $10 \mathrm{nA}$. The cut-off frequency of LPF is 0.75 times the bit rate. In the end, signal is completely recovered by 3R regenerator.

TX is mainly used in the pre-coding mode, which could not only increase the complexity of system, but also can be completely compatible with the conventional optical binary system [10]. Due to the difficulty of time delay in the high speed optical fiber transmission system, LPF is adopted to realize the electric double binary coding. The structure of LPF-ODB coding is shown in Figure 2 (TX). Firstly, the pseudo random binary sequence sends binary sequence to be pre-coding. In order to avoid the single signal symbol transmission errors in the binary optical communication transmission system [11], pre-coding should been used in optical duo-binary [12]. Figure 1(b) of the waveform can be found, due to the double binary code in the use of LPF method, resulting in the waveform in the "0" code is not completely zero [13]. Through the observation of Figure 3(c) radio spectrum, the ability of side lobe power suppression is poor.

The eye diagram and BER of four different kinds of dispersion compensation schemes are shown in Figure 4. Eye diagram reflects the changes of a signal waveform by overlapping every symbol waveform in a cycle. Comparison of Figures 4(a)-(d) of the eye can be found, the quality of eye diagram becomes better, which reflects the transmission performance is gradually enhanced. Using $Q$ factor to determine the performance of system [14], the simulate value from BER analyzer are follow: In the pre-compensation scheme, the maximum Q factor achieved 19.6 when the optimum threshold is 0.5 periods, and the minimum BER is $1 \mathrm{e}-84.9$. In the post-compensation scheme, the maximum $\mathrm{Q}$ factor achieved 22.6 when the optimum threshold is 0.48 period, and the minimum BER is $1 \mathrm{e}^{-113.2}$. In the hybridcompensation (pre+ post) scheme, the maximum $\mathrm{Q}$ factor 


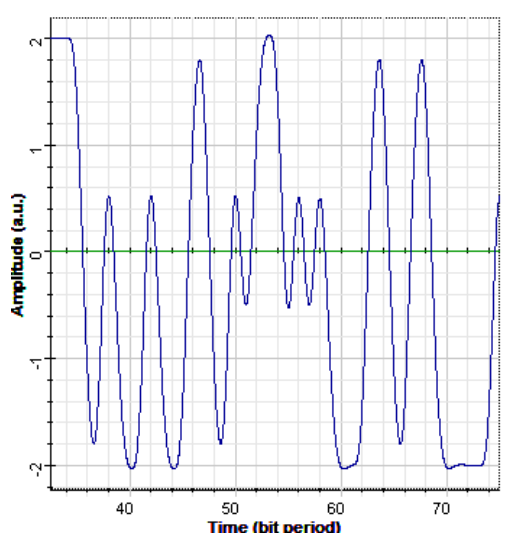

(a)

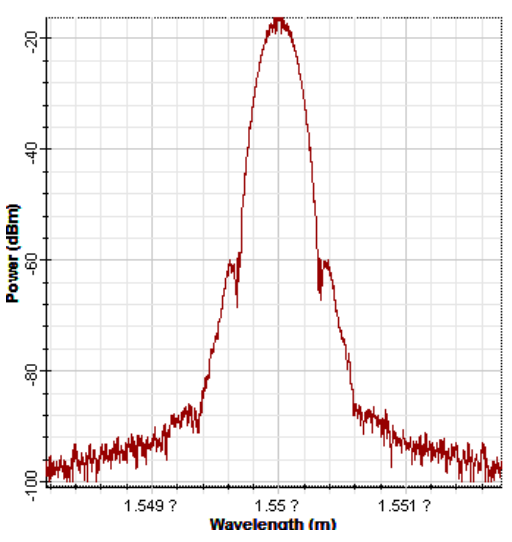

(b)

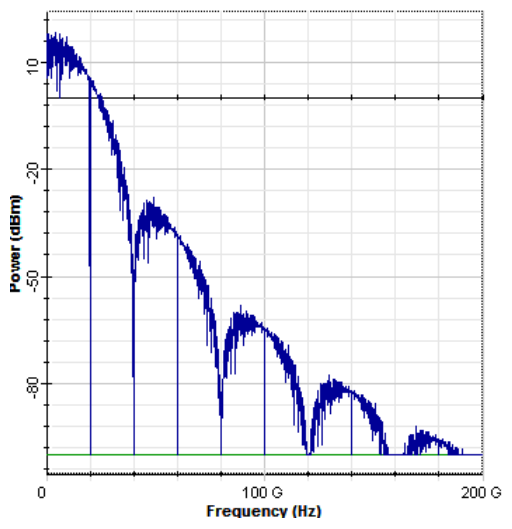

(c)

Figure 3. Diagrams of LPF-ODB (a) time domain wave (b) optical spectrum (c) electrical spectrum.

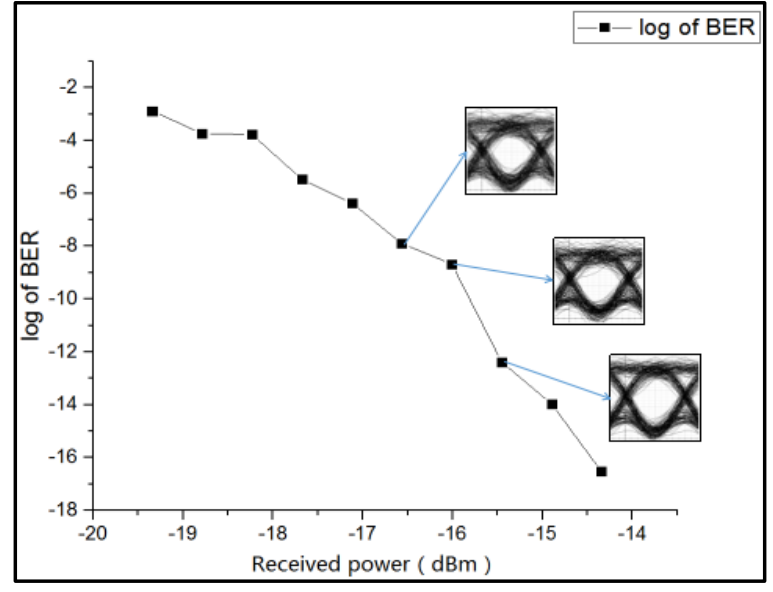

(a)

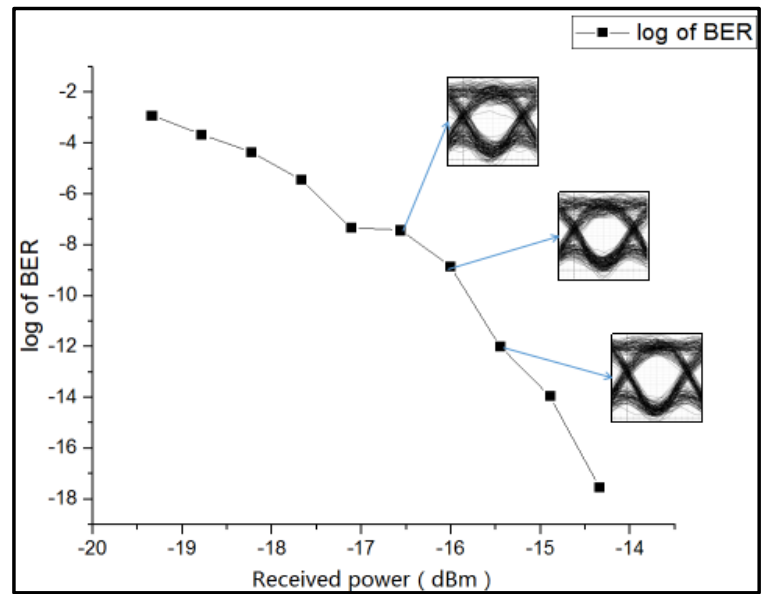

(c)

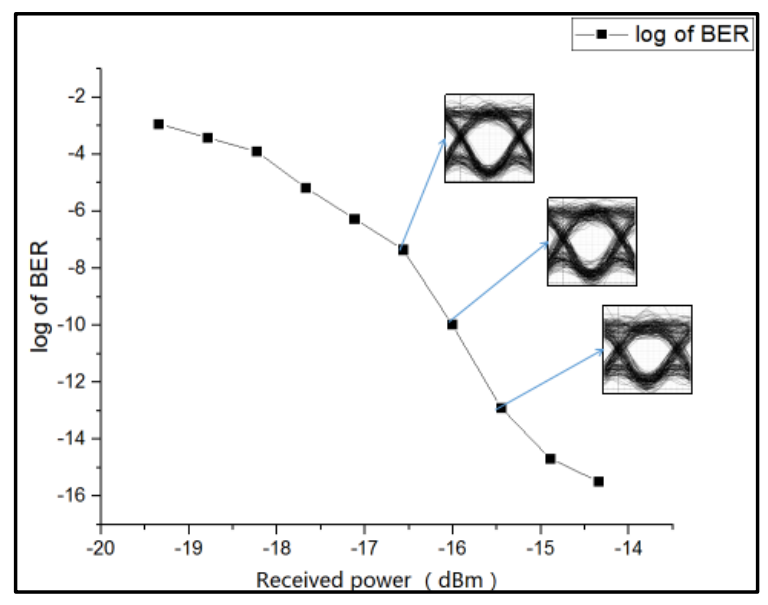

(b)

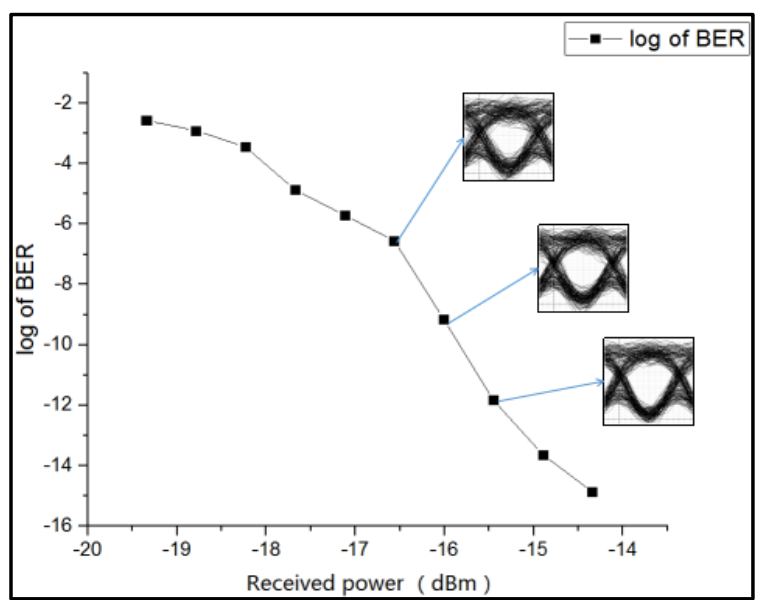

(d)

Figure 4. Four different dispersion compensation scheme transmission eye diagrams, and the BER curves (a) Pre-compensation (b) Post-compensation (c) Hybrid-compensation (pre+ post) (d) Hybrid-compensation (post+ pre).

achieved 25.4 when the optimum threshold is 0.5 period, and the minimum BER is $1 \mathrm{e}-142.5$. In the hybrid compensation (post+ pre) scheme, the maximum Q factor achieved 29.8 when the optimum threshold is 0.48 period, while the minimum BER is $1 \mathrm{e}-194.9$. By comparing the $\mathrm{Q}$ factor and BER, the transmission quality of the 
hybrid compensation (post + pre) is the best. Through the observation of eye diagram can be found, zero level has some disturbance because of LPF-ODB.

In order to scan the BER of different received power, an optical attenuator is added with the attenuation of 10 $-15 \mathrm{~dB}$ in simulation system. The diagram of the received power and BER is shown in Figure 4. According to the $\mathrm{Q}$ factor $=6$ and $\mathrm{BER}=1 \mathrm{e}-9$ as boundary conditions, compared the transport eye diagrams when $\mathrm{BER}=$ $1 \mathrm{e}^{-9}, 1 \mathrm{e}^{-}-8$, and $1 \mathrm{e}^{-10}$. When the $\mathrm{BER}=1 \mathrm{e}^{-9}$, the received power of pre-compensation and post-compensation both are $-15.9 \mathrm{~d} \mathrm{Bm}$, while hybrid compensation (pre+ post) and hybrid compensation (post + pre) respectively are $-16 \mathrm{dBm}$ and $-16.3 \mathrm{dBm}$. Obviously, hybrid compensation (post + pre) has the minimum received power and the highest sensitivity.

In this paper, the influence of the cut-off frequency of the LPF on the transmission performance is also analyzed. As shown in the Figure 5, the cut-off frequency of the low-pass filter can be adjusted from $12 \mathrm{GHz}$ to 18 $\mathrm{GHz}$, and the image of Q factor increased firstly and then decreased. When the cut-off frequency is $15 \mathrm{GHz}$, the $\mathrm{Q}$ factor reached the highest value of 29.7, the eye diagram is the most clear, with the optimal transmission performance. At the same time, it can be seen that with the increase of the cut-off frequency, the zero level eye disturbance increased either.

\section{Conclusion}

A $40 \mathrm{Gbit} / \mathrm{s}$ high speed optical fiber transmission system with different dispersion compensation schemes is proposed in this paper, which based on LPF-ODB coded modulation and combinations of DCF, SMF and EDFA. At the same time, the transmission performance influenced by the cut-off frequency of the LPF is also analyzed, and the optimum transmission performance can be achieved when the cut-off frequency is $15 \mathrm{GHz}$. By comparing the Q factor, BER, eye diagram and receive sensitivity of dispersion compensation schemes, it is found that the $\mathrm{Q}$ factor of hybrid compensation (post+ pre) is of the best project, which has the lowest BER and the highest receiving sensitivity. Finally, it is determined that using the transmission performance of hybrid compensation (post+ pre) scheme is optimal.

\section{Acknowledgements}

This work is partially supported by the National Natural Science Foundation of China (No. 61107064), Innovation Program of Shanghai Municipal Education Commission (No. 15ZZ101), Leading Academic Discipline Project of Information and Communication Engineering (No. XXKZD1605), School Foundation (No. EGD14XQD01) of Shanghai Polytechnic University, College Students' Science and Technology Innovation Project of Shanghai

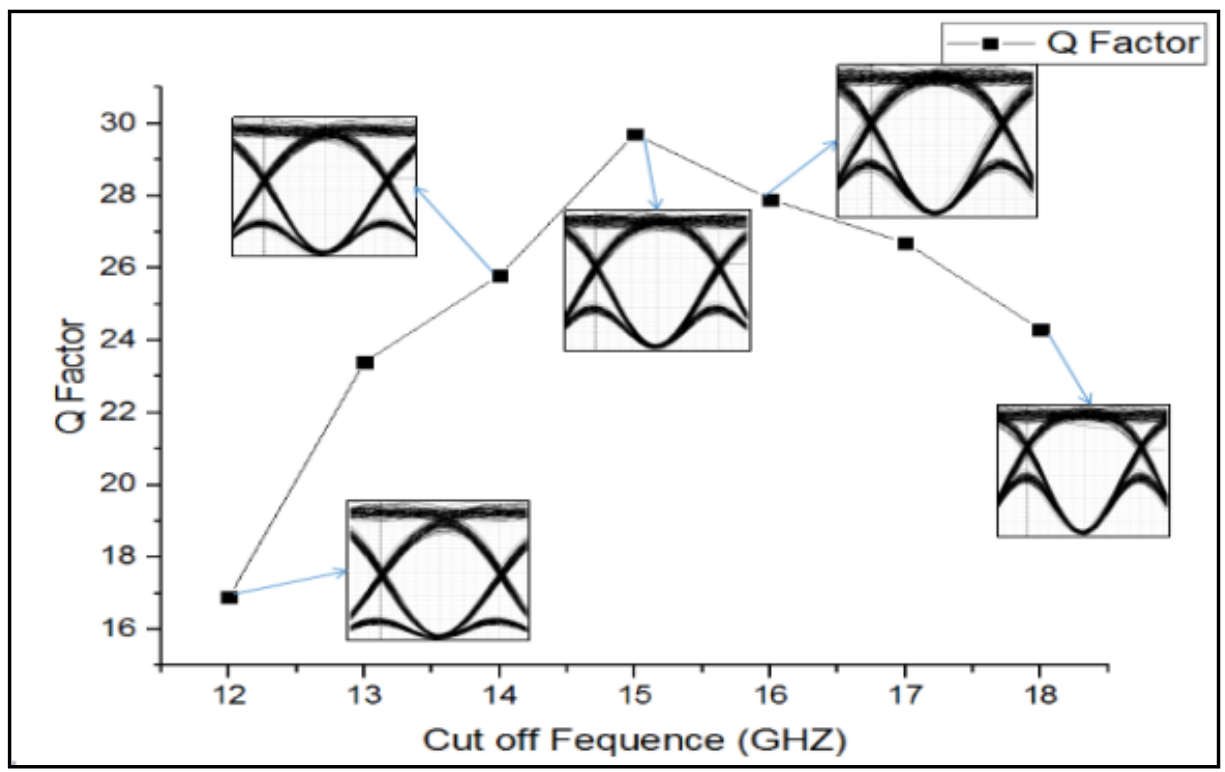

Figure 5. Q factor vs cut-off frequency of LPF. 
Polytechnic University (No. 2016-xjkj-063), and College Student Innovation Activity Plan in Shanghai (No. 2013-sj-cxjh-028).

\section{References}

[1] Li, J. (2007) A Dispersion Compensation Structure Based on Dual-Cavity GT Interferometer. Huazhong University of Science \&Technology, Wuhan. (In Chinese)

[2] Tokle, T. and Peucheret, C. (2003) Advanced Modulation Formats in 40Gbit/s Optical Communication Systems with 80km FibreSpans. Research Center Com, Technical University of Denmark.

[3] Cao, X. (2014) Optimization of Dispersion Compensation Scheme for Optical Fiber Communication System. Laser Technology, 38, 101-104.

[4] Zou, Y.Q. (2012) Simulation of Different Compensation Methods of Dispersion Compensation Fiber. Optical Instruments, 34, 11-16.

[5] Lyubomirsky, I. and Pitchumani, B. (2004) Impact of Optical Filtering Onduo-Binarytransmission. Photonics Technology Letters, IEEE, 16, 1969-1971. http://dx.doi.org/10.1109/LPT.2004.829751

[6] Fan, C.X., Zhan, D.Y., Xu, B.X., et al. (1995) Communications Principles. Beijing: Defense Industry Press.

[7] Li, Z.G. and Qiu, K. (2002) 40 GB/s Single Channel Optical Fiber Transmission System. University of Electronic Science and Technology, 5, 441-446.

[8] Zhou, Z.Q., Tang, Y.L. and Xie, C.J. (2000) Optimum Schemes of Dispersion Compensation Transmission Systems Using Dispersion Compensation Fibers. Laser Technology, 24, 265-269. (In Chinese)

[9] Pan, Q. and Wen, A.J. (2006) Comparison in 40Gb/s Single Channel RZ Modulation Formats Optical Transmission Dispersion Compensating System. Optical Communication Technology, 30, 61-62. (In Chinese)

[10] Ono, T., Yano, Y., Fukuchi, K., et al. (1998) Characteristics of Optical Duo-Binary Signals in Terabit/s Capacity, High-Spectral Efficiency WDM Systems. Journal of Lightwave Technology, 16, 788. http://dx.doi.org/10.1109/50.669006

[11] Ramaswami, R. and Sivrajan, K.N. (2004) Optical Networks: A Practical Perspective. 2nd Edition. China Machine Press, Beijing.

[12] Shao, Y.F., Chen, L. and Zhu, J.Z. (2007) A New Modified Decoding Scheme of Optical Duo-Binary Transmission. Journal of Communication, 2, 58-63, 74.

[13] Lu, Z.J., Wang, X.B. and Li, L. (2015) The Performance Analysis of Optical Double Binary Transmission in High Speed Optical Fiber System. Optical Communication Technology, 2, 34-37.

[14] Yu, J.J., Guan, K.J. and Yang, B.J. (1999) Numerical Simulation of a 10 Gbit/s Single Channel Long Distance Optical Communication System. Optical Communication Research, 1, 7-14, 18.

\section{Submit or recommend next manuscript to SCIRP and we will provide best service for you:}

Accepting pre-submission inquiries through Email, Facebook, Linkedin, Twitter, etc

A wide selection of journals (inclusive of 9 subjects, more than 200 journals)

Providing a 24-hour high-quality service

User-friendly online submission system

Fair and swift peer-review system

Efficient typesetting and proofreading procedure

Display of the result of downloads and visits, as well as the number of cited articles

Maximum dissemination of your research work

Submit your manuscript at: http://papersubmission.scirp.org/ 